\title{
Studies on Synthesis of Some Novel Heterocyclic Azlactone Derivatives and Imidazolinone Derivatives and their Antimicrobial Activity
}

\author{
RAKESH N. MISTRY and K. R. DESAI* \\ Department of Chemistry, \\ Veer Narmad South Gujarat University, \\ Surat - 395 007, GUJARAT (India).
}

Received 5 November 2004; Accepted 14 December 2004

\begin{abstract}
Methyl benzoic acid on reaction with phosphorus pentachloride gives $\mathrm{p}$ - methyl benzoyl chloride derivative which on condensation with glycine gives $\mathrm{p}$ methyl benzoyl glycine derivative. Now, this $\mathrm{p}$ - methyl benzoyl glycine derivative on condensation with various substituted aldehydes gives corresponding substituted 4 [aryl methylidine] - 2 - [p - methyl phenyl] - oxazole - 5 - one derivatives [1(a-j)]. Further, these derivatives $[1(\mathrm{a}-\mathrm{j})]$ on condensation with $4,4^{\prime}$ - diamino diphenyl sulphone gives corresponding substituted imidazolinone - dibenzsulphone derivatives [2(a-j)], on condensation with 4, 4' - diamino diphenyl methane gives corresponding substituted imidazolinone - dibenzmethane derivatives [3(a-j)], on condensation with 4,4'- diamino benzanilide gives corresponding substituted imidazolinone - benzanilide derivatives $[\mathbf{4}(\mathbf{a}-\mathbf{j})]$ and on condensation with 2 - amino pyridine gives corresponding substituted imidazolinone - pyridine derivatives $[\mathbf{5}(\mathbf{a}-\mathbf{j})]$ respectively. Structure elucidation of synthesised compounds has been made on the basis of elemental analysis, I.R. spectral studies and ${ }^{1}$ H N.M.R. spectral studies. The antimicrobial activity of the synthesised compounds has been studied against the cultures "Staphylococcus aureus", "Escherichia coli" and "Candela albicans".
\end{abstract}

Key words: Heterocyclic substituted oxazolone derivatives, imidazolinone - dibenzsulphone derivatives, imidazolinone - dibenzmethane derivatives, imidazolinone - benzanilide derivatives, imidazolinone - pyridine derivatives, antimicrobial activity. 


\section{Introduction}

The study incorporates the topic "AZLACTONE" because it provides a basic skeleton structure and which is also a part of a great importance for its drug Characteristics. The basic nucleus imidazole emerges from the drug intermediate azlactone. The azlactones possess oxazolone moiety. The azlactones are known to exhibit antifungal ${ }^{1}$, antibacterial ${ }^{2}$ and anti-inflammatory activities. They are also of great importance to produce penicillin type of drug intermediates ${ }^{3}$ and they are also useful to produce synthetic hormonal compounds ${ }^{4-5}$.

Imidazole is a planer five-membered heterocyclic ring system with three carbon and two nitrogen atoms in 1 and 3 positions. Imidazolones are keto dihydro imidazoles. Imidazolone that is known as oxoimidazoline is a five- membered heterocyclic ring system having nitrogen atoms in 1 and 3 positions and carbonyl group in 5 position. Oxoimidazoline, which is also known as imidazolinone is reported to exhibit a wide variety of therapeutic activities such as sedative, hypnotic, CNS depressant ${ }^{6}$ etc. Imidazolinone derivatives have also been reported to possess antihistaminic ${ }^{7}$, antihypertensive ${ }^{8}$ and antiparkinsonian' activities.

All these observations and the essential role of heterocyclic azlactone derivatives and imidazolinone derivatives, in certain biological reactions prompted us to synthesise all these heterocyclic derivatives $[\mathbf{1}(\mathbf{a}-\mathbf{j})$ to $\mathbf{5}(\mathbf{a}-\mathbf{j})]$.

\section{Experimental}

\section{Preparation of - Methyl Benzoyl Chloride Derivative}

A mixture of $p$ - methyl benzoic acid $(0.10 \mathrm{~mol})$ and phosphorus pentachloride $(0.12 \mathrm{~mol})$ was placed in a round - bottomed flask and the reaction mixture was refluxed in an oil bath at $120-130^{\circ} \mathrm{c}$ gently for about 2-3 hours. The reaction mixture was then allowed to cool and the phosphorus oxychloride was removed by distillation. The temperature of an oil bath was raised again up to $110^{\circ} \mathrm{c}$ and the residual $p$ - methyl benzoyl chloride was solidified on cooling, which was recrystallised from carbon tetrachloride. M. P. $108^{\circ}$ C., Yield $85 \%$.

\section{Preparation of p - Methyl Benzoyl Glycine Derivative-}

A glycine $(0.10 \mathrm{~mol})$ was dissolved in a $100 \mathrm{ml}$ of $10 \%$ sodium hydroxide solution and to it, $\mathrm{p}$ - methyl benzoyl chloride $(0.12 \mathrm{~mol})$ was added portion-wise and the reaction mixture was shaked vigorously after each addition until all the chloride has been reacted. The reaction mixture was then poured over crushed ice and acidified with concentrated $\mathrm{HCl}$ with constant stirring until the reaction mixture was acidic to Congo red paper. The resulting precipitate of $\mathrm{p}$ - methyl benzoyl glycine so obtained was filtered, washed several times with cold distilled water, dried and crystallised from carbon tetrachloride. M. P. $184^{\circ}$ C., Yield $76 \%$.

Preparation of 4 - [Phenyl Methylidine] - 2 - [p - Methyl Phenyl] - Oxazole - 5 - One Derivative [1(a)]

A mixture of benzaldehyde $(0.01 \mathrm{~mol}), \mathrm{p}$ - methyl benzoyl glycine $(0.01 \mathrm{~mol})$, acetic anhydride $(0.03$ mol) and anhydrous sodium acetate $(0.01 \mathrm{~mol})$ was taken in a $500 \mathrm{ml}$. conical flask and the reaction mixture was heated on an electric hotplate with constant shaking. As soon as the reaction mixture has been liquefied completely, the conical flask was transfered to a water bath and heated for about 2-3 hours. Then ethanol $(50 \mathrm{ml})$ was added slowly to the contents of the conical flask and the reaction mixture was allowed to stand overnight. The resulting precipitate so obtained was filtered, washed with ice-cold ethanol $(25 \mathrm{ml})$ and then with boiling water, dried and crystallised from benzene. M. P. $166^{\circ} \mathrm{C}$., Yield $65 \%$.

Similarly, the remaining substituted oxazolone derivatives $[\mathbf{1}(\mathbf{b}-\mathbf{j})]$ were prepared by the same procedure as discussed above.

Physical and Analytical data of compounds [1(a-j)] are presented in Table-1 and Antimicrobial data of compounds $[1(\mathrm{a}-\mathrm{j})]$ are presented in Table-6. 
Preparation of 4 4' - Bis - [2" - (p - Methyl Phenyl) - 4" - (Phenyl Methylidine) - Imidazole - 5" - One] - 1, 1'- Dibenzsulphone Derivative [2(a)]

A mixture of 4 - [phenyl methylidine] - 2 - [p - methyl phenyl] - oxazole - 5 - one [1(a)] (0.02 mol) and 4,4 ' - diamino diphenyl sulphone $(0.01 \mathrm{~mol})$ was dissolved in a dry pyridine $(25 \mathrm{ml})$ and the reaction mixture was refluxed in an oil bath at $110-120^{\circ} \mathrm{c}$ gently for about 5-6 hours. After the completion of reaction, the reaction mixture was poured over crushed ice and neutralised with dilute $\mathrm{HCl}$. The resulting solid so obtained was filtered, washed several times with distilled water, dried and crystallised from DMSO. M. P. $187^{\circ}$ C., Yield $78 \%$.

Similarly, the remaining substituted imidazolinone - dibenzsulphone derivatives [2(b-j)] were prepared by the same procedure as discussed above.

Physical and Analytical data of compounds [2(a-j)] are presented in Table-2 and Antimicrobial data of compounds $[2(\mathrm{a}-\mathrm{j})]$ are presented in Table -7 .

Preparation Of 4 , 4' - Bis - [2" - ( $p$ - Methyl Phenyl) - 4” - (Phenyl Methylidine) - Imidazole 5" - One] - 1, 1' - Dibenzmethane Derivative [3(a)]

A mixture of 4 - [phenyl methylidine] - 2 - [p - methyl phenyl] - oxazole - 5 - one [1(a)] (0.02 mol) and 4,4 ' - diamino diphenyl methane $(0.01 \mathrm{~mol})$ was dissolved in a dry pyridine $(25 \mathrm{ml})$ and the reaction mixture was refluxed in an oil bath at $110-120^{\circ} \mathrm{c}$ gently for about 5-6 hours. After the completion of reaction, the reaction mixture was poured over crushed ice and neutralised with dilute $\mathrm{HCl}$. The resulting solid so obtained was filtered, washed several times with distilled water, dried and crystallised from DMSO. M. P. $182^{\circ}$ C., Yield $73 \%$.

Similarly, the remaining substituted imidazolinone - dibenzmethane derivatives $[\mathbf{3}(\mathbf{b}-\mathbf{j})]$ were prepared by the same procedure as discussed above.

Physical and Analytical data of compounds [3(a-j)] are presented in Table $\mathbf{- 3}$ and Antimicrobial data of compounds $[3(\mathrm{a}-\mathrm{j})]$ are presented in Table $\mathbf{- 8}$.

Preparation of 4 4' - Bis - [2" - (p - Methyl Phenyl) - 4" - (Phenyl Methylidine) - Imidazole - 5" - One] - 1, 1'- Benzanilide Derivative [4(a)]

A mixture of 4 - [phenyl methylidine] - 2 - [p - methyl phenyl] - oxazole - 5 - one [1(a)] (0.02 mol) and $4,4^{\prime}$ - diamino benzanilide $(0.01 \mathrm{~mol})$ was dissolved in a dry pyridine $(25 \mathrm{ml})$ and the reaction mixture was refluxed in an oil bath at $110-120^{\circ} \mathrm{c}$ gently for about 5-6 hours. After the completion of reaction, the reaction mixture was poured over crushed ice and neutralised with dilute $\mathrm{HCl}$. The resulting solid so obtained was filtered, washed several times with distilled water, dried and crystallised from DMSO. M. P. $173^{\circ}$ C., Yield $70 \%$.

Similarly, the remaining substituted imidazolinone - benzanilide derivatives $[\mathbf{4}(\mathbf{b}-\mathbf{j})]$ were prepared by the same procedure as discussed above.

Physical and Analytical data of compounds [4(a-j)] are presented in Table $\mathbf{- 4}$ and Antimicrobial data of compounds $[4(\mathrm{a}-\mathrm{j})]$ are presented in Table -9.

Preparation of 1 - Pyridine - 2 - [p - Methyl Phenyl] - 4 - [Phenyl Methylidine] - Imidazole - 5 - One Derivative [5(a)]

A mixture of 4 - [phenyl methylidine] - 2 - [p - methyl phenyl] - oxazole - 5 - one [1(a)] (0.01 mol) and 2 - amino pyridine $(0.01 \mathrm{~mol})$ was dissolved in a dry pyridine $(25 \mathrm{ml})$ and the reaction mixture was refluxed in an oil bath at $110-120^{\circ} \mathrm{c}$ gently for about 5-6 hours. After the completion of reaction, the reaction mixture was poured over crushed ice and neutralised with dilute $\mathrm{HCl}$. The resulting solid so obtained was filtered, washed several times with distilled water, dried and crystallised from DMSO. M. P. $192^{\circ}$ C., Yield $77 \%$.

Similarly, the remaining substituted imidazolinone - pyridine derivatives $[\mathbf{5}(\mathbf{b}-\mathbf{j})]$ were prepared by the same procedure as discussed above.

Physical and Analytical data of compounds [5(a-j)] are presented in Table -5 and Antimicrobial data of compounds [5(a-j)] are presented in Table $\mathbf{- 1 0}$. 
Table -1. PHYSICAL AND ANALYTICAL DATA OF COMPOUNDS [1(a-j)]

\begin{tabular}{|c|c|c|c|c|c|c|c|}
\hline \multirow{2}{*}{ Compd. No. } & \multirow{2}{*}{$\mathrm{R}$} & \multirow{2}{*}{$\begin{array}{c}\text { M. F. } \\
\text { [M. W. }(\mathrm{g} / \mathrm{m})]\end{array}$} & \multirow{2}{*}{$\begin{array}{c}\text { M. } \\
\text { P. } \\
\left({ }^{\circ} \mathrm{C}\right)\end{array}$} & \multirow{2}{*}{$\begin{array}{l}\text { Yield } \\
(\%)\end{array}$} & \multicolumn{3}{|c|}{$\begin{array}{c}\% \text { Analysis } \\
\text { (Calcd.) }\end{array}$ Found } \\
\hline & & & & & C \% & H \% & $\mathbf{N} \%$ \\
\hline 1 a. & $-\mathrm{H}$ & $\begin{array}{c}\mathrm{C}_{17} \mathrm{H}_{13} \mathrm{NO}_{2} \\
(263.0)\end{array}$ & 166 & 65 & $\begin{array}{c}77.54 \\
(77.57)\end{array}$ & $\begin{array}{c}4.92 \\
(4.94)\end{array}$ & $\begin{array}{c}5.30 \\
(5.32)\end{array}$ \\
\hline $1 \mathrm{~b}$. & $2-0 \mathrm{H}$ & $\begin{array}{c}\mathrm{C}_{17} \mathrm{H}_{13} \mathrm{NO}_{3} \\
(279.0)\end{array}$ & 174 & 68 & $\begin{array}{l}73.10 \\
(73.12)\end{array}$ & $\begin{array}{c}4.63 \\
(4.66)\end{array}$ & $\begin{array}{c}5.00 \\
(5.02)\end{array}$ \\
\hline $1 \mathrm{c}$. & $2-\mathrm{NO}_{2}$ & $\begin{array}{l}\mathrm{C}_{17} \mathrm{H}_{12} \mathrm{~N}_{2} \mathrm{O}_{4} \\
(308.0)\end{array}$ & 168 & 64 & $\begin{array}{c}66.20 \\
(66.23)\end{array}$ & $\begin{array}{c}3.86 \\
(3.90)\end{array}$ & $\begin{array}{c}9.06 \\
(9.09)\end{array}$ \\
\hline $1 \mathrm{~d}$. & 2-CI & $\begin{array}{c}\mathrm{C}_{17} \mathrm{H}_{12} \mathrm{NO}_{2} \mathrm{CI} \\
(297.5)\end{array}$ & 155 & 70 & $\begin{array}{l}68.55 \\
(68.57)\end{array}$ & $\begin{array}{c}4.00 \\
(4.03)\end{array}$ & $\begin{array}{c}4.67 \\
(4.70)\end{array}$ \\
\hline 1 e. & $3-\mathrm{Br}$ & $\begin{array}{c}\mathrm{C}_{17} \mathrm{H}_{12} \mathrm{NO}_{2} \mathrm{Br} \\
(342.0)\end{array}$ & 158 & 66 & $\begin{array}{c}59.64 \\
(59.65)\end{array}$ & $\begin{array}{c}3.49 \\
(3.51)\end{array}$ & $\begin{array}{c}4.08 \\
(4.09)\end{array}$ \\
\hline $1 \mathrm{f}$. & 4-CI & $\begin{array}{c}\mathrm{C}_{17} \mathrm{H}_{12} \mathrm{NO}_{2} \mathrm{CI} \\
(297.5)\end{array}$ & 150 & 72 & $\begin{array}{c}68.53 \\
(68.57)\end{array}$ & $\begin{array}{c}4.00 \\
(4.03)\end{array}$ & $\begin{array}{c}4.67 \\
(4.70)\end{array}$ \\
\hline $1 \mathrm{~g}$. & $2-\mathrm{OCH}_{3}$ & $\begin{array}{l}\mathrm{C}_{18} \mathrm{H}_{15} \mathrm{NO}_{3} \\
(293.0)\end{array}$ & 170 & 75 & $\begin{array}{c}73.70 \\
(73.72)\end{array}$ & $\begin{array}{c}5.09 \\
(5.12)\end{array}$ & $\begin{array}{c}4.76 \\
(4.78)\end{array}$ \\
\hline $1 \mathrm{~h}$. & $\begin{array}{c}3,4- \\
\left(\mathrm{OCH}_{3}\right)_{2}\end{array}$ & $\begin{array}{c}\mathrm{C}_{19} \mathrm{H}_{17} \mathrm{NO}_{4} \\
(323.0)\end{array}$ & 152 & 68 & $\begin{array}{c}70.55 \\
(70.59)\end{array}$ & $\begin{array}{l}5.25 \\
(5.26)\end{array}$ & $\begin{array}{c}4.29 \\
(4.33)\end{array}$ \\
\hline $1 \mathrm{i}$. & $4-\mathrm{OCH}_{3}$ & $\begin{array}{c}\mathrm{C}_{18} \mathrm{H}_{15} \mathrm{NO}_{3} \\
(293.0)\end{array}$ & 167 & 70 & $\begin{array}{c}73.69 \\
(73.72)\end{array}$ & $\begin{array}{c}5.10 \\
(5.12)\end{array}$ & $\begin{array}{c}4.75 \\
(4.78)\end{array}$ \\
\hline $1 \mathrm{j}$. & $\begin{array}{l}3,4,5- \\
\left(\mathrm{OCH}_{3}\right)_{3}\end{array}$ & $\begin{array}{c}\mathrm{C}_{20} \mathrm{H}_{19} \mathrm{NO}_{5} \\
(353.0)\end{array}$ & 173 & 66 & $\begin{array}{l}67.97 \\
(67.99)\end{array}$ & $\begin{array}{c}5.35 \\
(5.38)\end{array}$ & $\begin{array}{c}3.95 \\
(3.97)\end{array}$ \\
\hline
\end{tabular}

Table - 2 PHYSICAL AND ANALYTICAL DATA OF COMPOUNDS [2(a-j)]

\begin{tabular}{|c|c|c|c|c|c|c|c|}
\hline \multirow{2}{*}{$\begin{array}{l}\text { Compd. } \\
\text { No. }\end{array}$} & \multirow{2}{*}{$\mathrm{R}$} & \multirow{2}{*}{$\begin{array}{c}\text { M. F. } \\
{[\text { M. W. }(g / m)]}\end{array}$} & \multirow{2}{*}{$\begin{array}{l}\text { M. P. } \\
\left({ }^{\circ} \mathrm{C}\right)\end{array}$} & \multirow{2}{*}{$\begin{array}{l}\text { Yield } \\
(\%)\end{array}$} & \multicolumn{3}{|c|}{$\begin{array}{c}\% \text { Analysis } \\
\text { (Calcd.) }\end{array}$ Found } \\
\hline & & & & & C \% & H \% & $\mathbf{N} \%$ \\
\hline 2 a. & $-\mathrm{H}$ & $\mathrm{C}_{46} \mathrm{H}_{34} \mathrm{~N}_{4} \mathrm{O}_{4} \mathrm{~S}(738.0)$ & 188 & 74 & $\begin{array}{c}74.77 \\
(74.80)\end{array}$ & $\begin{array}{c}4.59 \\
(4.61)\end{array}$ & $\begin{array}{c}7.56 \\
(7.59)\end{array}$ \\
\hline $2 \mathrm{~b}$. & $2-0 \mathrm{H}$ & $\mathrm{C}_{46} \mathrm{H}_{34} \mathrm{~N}_{4} \mathrm{O}_{6} \mathrm{~S}(770.0)$ & 183 & 68 & $\begin{array}{l}71.67 \\
(71.69)\end{array}$ & $\begin{array}{c}4.37 \\
(4.41)\end{array}$ & $\begin{array}{c}7.25 \\
(7.27)\end{array}$ \\
\hline $2 \mathrm{c}$. & $2-\mathrm{NO}_{2}$ & $\mathrm{C}_{46} \mathrm{H}_{32} \mathrm{~N}_{6} \mathrm{O}_{8} \mathrm{~S}(828.0)$ & 170 & 70 & $\begin{array}{c}66.64 \\
(66.67)\end{array}$ & $\begin{array}{c}3.85 \\
(3.86)\end{array}$ & $\begin{array}{c}10.11 \\
(10.14)\end{array}$ \\
\hline $2 \mathrm{~d}$ & 2-CI & $\begin{array}{c}\mathrm{C}_{46} \mathrm{H}_{32} \mathrm{~N}_{4} \mathrm{O}_{4} \mathrm{SCI}_{2} \\
(807.0)\end{array}$ & 198 & 75 & $\begin{array}{l}68.36 \\
(68.40)\end{array}$ & $\begin{array}{c}3.93 \\
(3.96)\end{array}$ & $\begin{array}{c}6.91 \\
(6.94)\end{array}$ \\
\hline $2 \mathrm{e}$. & $3-\mathrm{Br}$ & $\begin{array}{c}\mathrm{C}_{46} \mathrm{H}_{32} \mathrm{~N}_{4} \mathrm{O}_{4} \mathrm{SBr}_{2} \\
(896.0)\end{array}$ & 193 & 68 & $\begin{array}{c}61.60 \\
(61.61)\end{array}$ & $\begin{array}{c}3.55 \\
(3.57)\end{array}$ & $\begin{array}{c}6.21 \\
(6.25)\end{array}$ \\
\hline $2 \mathrm{f}$. & 4-CI & $\begin{array}{c}\mathrm{C}_{46} \mathrm{H}_{32} \mathrm{~N}_{4} \mathrm{O}_{4} \mathrm{SCI}_{2} \\
(807.0)\end{array}$ & 203 & 73 & $\begin{array}{c}68.37 \\
(68.40)\end{array}$ & $\begin{array}{c}3.93 \\
(3.96)\end{array}$ & $\begin{array}{c}6.91 \\
(6.94)\end{array}$ \\
\hline $2 \mathrm{~g}$. & $2-\mathrm{OCH}_{3}$ & $\mathrm{C}_{48} \mathrm{H}_{38} \mathrm{~N}_{4} \mathrm{O}_{6} \mathrm{~S}(798.0)$ & 168 & 72 & $\begin{array}{l}72.16 \\
(72.18)\end{array}$ & $\begin{array}{c}4.74 \\
(4.76)\end{array}$ & $\begin{array}{c}7.00 \\
(7.02)\end{array}$ \\
\hline $2 \mathrm{~h}$. & $\begin{array}{c}3,4- \\
\left(\mathrm{OCH}_{3}\right)_{2}\end{array}$ & $\mathrm{C}_{50} \mathrm{H}_{42} \mathrm{~N}_{4} \mathrm{O}_{8} \mathrm{~S}(858.0)$ & 185 & 66 & $\begin{array}{c}69.90 \\
(69.93)\end{array}$ & $\begin{array}{c}4.85 \\
(4.89)\end{array}$ & $\begin{array}{c}6.52 \\
(6.53)\end{array}$ \\
\hline $2 \mathrm{i}$. & $4-\mathrm{OCH}_{3}$ & $\mathrm{C}_{48} \mathrm{H}_{38} \mathrm{~N}_{4} \mathrm{O}_{6} \mathrm{~S}(798.0)$ & 176 & 76 & $\begin{array}{l}72.15 \\
(72.18)\end{array}$ & $\begin{array}{c}4.74 \\
(4.76)\end{array}$ & $\begin{array}{c}6.99 \\
(7.02)\end{array}$ \\
\hline $2 \mathrm{j}$. & $\begin{array}{l}3,4,5- \\
\left(\mathrm{OCH}_{3}\right)_{3}\end{array}$ & $\begin{array}{c}\mathrm{C}_{52} \mathrm{H}_{46} \mathrm{~N}_{4} \mathrm{O}_{10} \mathrm{~S} \\
(918.0)\end{array}$ & 208 & 70 & $\begin{array}{c}67.94 \\
(67.97)\end{array}$ & $\begin{array}{c}4.98 \\
(5.01)\end{array}$ & $\begin{array}{c}6.08 \\
(6.10)\end{array}$ \\
\hline
\end{tabular}


Table - 3 PHYSICAL AND ANALYTICAL DATA OF COMPOUNDS [3(a-j)]

\begin{tabular}{|c|c|c|c|c|c|c|c|}
\hline \multirow{2}{*}{$\begin{array}{l}\text { Compd. } \\
\text { No. }\end{array}$} & \multirow{2}{*}{$\mathrm{R}$} & \multirow{2}{*}{$\begin{array}{c}\text { M. F. } \\
{[\text { M. W. }(\mathrm{g} / \mathrm{m})]}\end{array}$} & \multirow{2}{*}{$\begin{array}{c}\text { M. P. } \\
\left({ }^{\circ} \mathrm{C}\right)\end{array}$} & \multirow{2}{*}{$\begin{array}{c}\text { Yield } \\
(\%)\end{array}$} & \multirow{2}{*}{$\frac{\% \text { Analysis }}{\mathrm{C} \%}$} & \multicolumn{2}{|c|}{ Found (Calcd.) } \\
\hline & & & & & & $\mathrm{H} \%$ & $\mathrm{~N} \%$ \\
\hline 3 a. & $-\mathrm{H}$ & $\begin{array}{c}\mathrm{C}_{47} \mathrm{H}_{36} \mathrm{~N}_{4} \mathrm{O}_{2} \\
(688.0)\end{array}$ & 180 & 76 & $\begin{array}{c}81.96 \\
(81.98)\end{array}$ & $\begin{array}{c}5.20 \\
(5.23)\end{array}$ & $\begin{array}{c}8.11 \\
(8.14)\end{array}$ \\
\hline 3 b. & $2-0 \mathrm{H}$ & $\begin{array}{c}\mathrm{C}_{47} \mathrm{H}_{36} \mathrm{~N}_{4} \mathrm{O}_{4} \\
(720.0)\end{array}$ & 187 & 67 & $\begin{array}{c}78.30 \\
(78.33)\end{array}$ & $\begin{array}{c}4.99 \\
(5.00)\end{array}$ & $\begin{array}{c}7.76 \\
(7.78)\end{array}$ \\
\hline $3 \mathrm{c}$. & $2-\mathrm{NO}_{2}$ & $\begin{array}{c}\mathrm{C}_{47} \mathrm{H}_{34} \mathrm{~N}_{6} \mathrm{O}_{6} \\
(778.0)\end{array}$ & 166 & 72 & $\begin{array}{c}72.45 \\
(72.49)\end{array}$ & $\begin{array}{c}4.35 \\
(4.37)\end{array}$ & $\begin{array}{c}10.77 \\
(10.80)\end{array}$ \\
\hline $3 \mathrm{~d}$. & 2-CI & $\begin{array}{c}\mathrm{C}_{47} \mathrm{H}_{34} \mathrm{~N}_{4} \mathrm{O}_{2} \mathrm{CI}_{2} \\
(757.0)\end{array}$ & 195 & 70 & $\begin{array}{c}74.47 \\
(74.50)\end{array}$ & $\begin{array}{c}4.45 \\
(4.49)\end{array}$ & $\begin{array}{c}7.38 \\
(7.40)\end{array}$ \\
\hline 3 e. & $3-\mathrm{Br}$ & $\begin{array}{c}\mathrm{C}_{47} \mathrm{H}_{34} \mathrm{~N}_{4} \mathrm{O}_{2} \mathrm{Br}_{2} \\
(846.0)\end{array}$ & 190 & 68 & $\begin{array}{c}66.66 \\
(66.67)\end{array}$ & $\begin{array}{c}4.00 \\
(4.02)\end{array}$ & $\begin{array}{c}6.58 \\
(6.62)\end{array}$ \\
\hline $3 \mathrm{f}$. & 4-CI & $\begin{array}{c}\mathrm{C}_{47} \mathrm{H}_{34} \mathrm{~N}_{4} \mathrm{O}_{2} \mathrm{CI}_{2} \\
(757.0)\end{array}$ & 205 & 74 & $\begin{array}{c}74.47 \\
(74.50)\end{array}$ & $\begin{array}{c}4.46 \\
(4.49)\end{array}$ & $\begin{array}{c}7.38 \\
(7.40)\end{array}$ \\
\hline $3 \mathrm{~g}$. & $2-\mathrm{OCH}_{3}$ & $\begin{array}{c}\mathrm{C}_{49} \mathrm{H}_{40} \mathrm{~N}_{4} \mathrm{O}_{4} \\
(748.0)\end{array}$ & 176 & 66 & $\begin{array}{c}78.59 \\
(78.61)\end{array}$ & $\begin{array}{c}5.32 \\
(5.35)\end{array}$ & $\begin{array}{c}7.48 \\
(7.49)\end{array}$ \\
\hline $3 \mathrm{~h}$. & $\begin{array}{c}3,4- \\
\left(\mathrm{OCH}_{3}\right)_{2}\end{array}$ & $\begin{array}{c}\mathrm{C}_{51} \mathrm{H}_{44} \mathrm{~N}_{4} \mathrm{O}_{6} \\
(808.0)\end{array}$ & 187 & 76 & $\begin{array}{c}75.73 \\
(75.74)\end{array}$ & $\begin{array}{c}5.43 \\
(5.44)\end{array}$ & $\begin{array}{c}6.90 \\
(6.93)\end{array}$ \\
\hline 3 i. & $4-\mathrm{OCH}_{3}$ & $\begin{array}{c}\mathrm{C}_{49} \mathrm{H}_{40} \mathrm{~N}_{4} \mathrm{O}_{4} \\
(748.0)\end{array}$ & 174 & 65 & $\begin{array}{c}78.58 \\
(78.61)\end{array}$ & $\begin{array}{c}5.32 \\
(5.35)\end{array}$ & $\begin{array}{c}7.47 \\
(7.49)\end{array}$ \\
\hline $3 \mathrm{j}$. & $\begin{array}{l}3,4,5- \\
\left(\mathrm{OCH}_{3}\right)_{3}\end{array}$ & $\begin{array}{c}\mathrm{C}_{53} \mathrm{H}_{48} \mathrm{~N}_{4} \mathrm{O}_{8} \\
(868.0)\end{array}$ & 214 & 68 & $\begin{array}{c}73.24 \\
(73.27) \\
\end{array}$ & $\begin{array}{r}5.50 \\
(5.53) \\
\end{array}$ & $\begin{array}{c}6.43 \\
(6.45) \\
\end{array}$ \\
\hline
\end{tabular}

Table - 4 PHYSICAL AND ANALYTICAL DATA OF COMPOUNDS [4(a-j)]

\begin{tabular}{|c|c|c|c|c|c|c|c|}
\hline \multirow{2}{*}{$\begin{array}{c}\text { Compd. } \\
\text { No. }\end{array}$} & \multirow{2}{*}{$\mathrm{R}$} & \multirow{2}{*}{$\begin{array}{c}\text { M. F. } \\
{[\text { M. W. }(\mathrm{g} / \mathrm{m})]}\end{array}$} & \multirow{2}{*}{$\begin{array}{l}\text { M. P. } \\
\left({ }^{\circ} \mathrm{C}\right)\end{array}$} & \multirow{2}{*}{$\begin{array}{c}\text { Yield } \\
(\%)\end{array}$} & \multirow{2}{*}{$\frac{\% \text { Analysis }}{\mathrm{C} \%}$} & \multicolumn{2}{|c|}{ Found (Calcd.) } \\
\hline & & & & & & $\mathrm{H} \%$ & $\mathrm{~N} \%$ \\
\hline 4 a. & $-\mathrm{H}$ & $\begin{array}{c}\mathrm{C}_{47} \mathrm{H}_{35} \mathrm{~N}_{5} \mathrm{O}_{3} \\
(717.0)\end{array}$ & 178 & 75 & $\begin{array}{c}78.63 \\
(78.66)\end{array}$ & $\begin{array}{c}4.86 \\
(4.88)\end{array}$ & $\begin{array}{c}9.73 \\
(9.76)\end{array}$ \\
\hline $4 \mathrm{~b}$. & $2-0 \mathrm{H}$ & $\begin{array}{c}\mathrm{C}_{47} \mathrm{H}_{35} \mathrm{~N}_{5} \mathrm{O}_{5} \\
(749.0)\end{array}$ & 184 & 68 & $\begin{array}{l}75.28 \\
(75.30)\end{array}$ & $\begin{array}{c}4.64 \\
(4.67)\end{array}$ & $\begin{array}{c}9.30 \\
(9.34)\end{array}$ \\
\hline $4 \mathrm{c}$ & $2-\mathrm{NO}_{2}$ & $\begin{array}{c}\mathrm{C}_{47} \mathrm{H}_{33} \mathrm{~N}_{7} \mathrm{O}_{7} \\
(807.0)\end{array}$ & 172 & 64 & $\begin{array}{c}69.85 \\
(69.89)\end{array}$ & $\begin{array}{c}4.07 \\
(4.09)\end{array}$ & $\begin{array}{l}12.13 \\
(12.14)\end{array}$ \\
\hline $4 \mathrm{~d}$. & 2-CI & $\begin{array}{c}\mathrm{C}_{47} \mathrm{H}_{33} \mathrm{~N}_{5} \mathrm{O}_{3} \mathrm{CI}_{2} \\
(786.0)\end{array}$ & 198 & 72 & $\begin{array}{c}71.72 \\
(71.75)\end{array}$ & $\begin{array}{c}4.17 \\
(4.20)\end{array}$ & $\begin{array}{c}8.87 \\
(8.90)\end{array}$ \\
\hline 4 e. & $3-\mathrm{Br}$ & $\begin{array}{c}\mathrm{C}_{47} \mathrm{H}_{33} \mathrm{~N}_{5} \mathrm{O}_{3} \mathrm{Br}_{2} \\
(875.0)\end{array}$ & 186 & 70 & $\begin{array}{c}64.42 \\
(64.46)\end{array}$ & $\begin{array}{c}3.74 \\
(3.77)\end{array}$ & $\begin{array}{l}7.97 \\
(8.00)\end{array}$ \\
\hline $4 \mathrm{f}$. & 4-CI & $\begin{array}{c}\mathrm{C}_{47} \mathrm{H}_{33} \mathrm{~N}_{5} \mathrm{O}_{3} \mathrm{CI}_{2} \\
(786.0)\end{array}$ & 207 & 75 & $\begin{array}{l}71.73 \\
(71.75)\end{array}$ & $\begin{array}{c}4.17 \\
(4.20)\end{array}$ & $\begin{array}{c}8.88 \\
(8.90)\end{array}$ \\
\hline $4 \mathrm{~g}$. & $2-\mathrm{OCH}_{3}$ & $\begin{array}{c}\mathrm{C}_{49} \mathrm{H}_{39} \mathrm{~N}_{5} \mathrm{O}_{5} \\
(777.0)\end{array}$ & 180 & 72 & $\begin{array}{c}75.65 \\
(75.67)\end{array}$ & $\begin{array}{c}5.00 \\
(5.02)\end{array}$ & $\begin{array}{c}8.97 \\
(9.00)\end{array}$ \\
\hline $4 \mathrm{~h}$. & $\begin{array}{c}3,4- \\
\left(\mathrm{OCH}_{3}\right)_{2}\end{array}$ & $\begin{array}{c}\mathrm{C}_{51} \mathrm{H}_{43} \mathrm{~N}_{5} \mathrm{O}_{7} \\
(837.0)\end{array}$ & 188 & 64 & $\begin{array}{l}73.11 \\
(73.12)\end{array}$ & $\begin{array}{c}5.13 \\
(5.14)\end{array}$ & $\begin{array}{c}8.34 \\
(8.36)\end{array}$ \\
\hline $4 \mathrm{i}$. & $4-\mathrm{OCH}_{3}$ & $\begin{array}{c}\mathrm{C}_{49} \mathrm{H}_{39} \mathrm{~N}_{5} \mathrm{O}_{5} \\
(777.0)\end{array}$ & 173 & 67 & $\begin{array}{l}75.65 \\
(75.67)\end{array}$ & $\begin{array}{c}5.00 \\
(5.02)\end{array}$ & $\begin{array}{c}8.97 \\
(9.00)\end{array}$ \\
\hline $4 \mathrm{j}$. & $\begin{array}{l}3,4,5- \\
\left(\mathrm{OCH}_{3}\right)_{3}\end{array}$ & $\begin{array}{c}\mathrm{C}_{53} \mathrm{H}_{47} \mathrm{~N}_{5} \mathrm{O}_{9} \\
(897.0)\end{array}$ & 210 & 65 & $\begin{array}{c}70.87 \\
(70.90)\end{array}$ & $\begin{array}{c}5.22 \\
(5.24)\end{array}$ & $\begin{array}{c}7.78 \\
(7.80)\end{array}$ \\
\hline
\end{tabular}


Table - 5 PHYSICAL AND ANALYTICAL DATA OF COMPOUNDS [5(a-j)]

\begin{tabular}{|c|c|c|c|c|c|c|c|}
\hline \multirow{2}{*}{$\begin{array}{l}\text { Compd. } \\
\text { No. }\end{array}$} & \multirow{2}{*}{$\mathrm{R}$} & \multirow{2}{*}{$\begin{array}{l}\text { M. F. } \\
\text { W. }(\mathrm{g} / \mathrm{m})]\end{array}$} & \multirow{2}{*}{$\begin{array}{l}\text { M. P. } \\
\left({ }^{\circ} \mathrm{C}\right)\end{array}$} & \multirow{2}{*}{$\begin{array}{l}\text { Yield } \\
(\%)\end{array}$} & \multirow{2}{*}{$\frac{\% \text { Analysis }}{\mathrm{C} \%}$} & \multicolumn{2}{|c|}{ Found (Calcd.) } \\
\hline & & & & & & $\mathrm{H} \%$ & $\mathrm{~N} \%$ \\
\hline 5 a. & $-\mathrm{H}$ & $\begin{array}{c}\mathrm{C}_{22} \mathrm{H}_{17} \mathrm{~N}_{3} \mathrm{O} \\
(339.0)\end{array}$ & 192 & 77 & $\begin{array}{c}77.86 \\
(77.88)\end{array}$ & $\begin{array}{c}5.00 \\
(5.01)\end{array}$ & $\begin{array}{c}12.36 \\
(12.39)\end{array}$ \\
\hline $5 \mathrm{~b}$. & $2-0 \mathrm{H}$ & $\begin{array}{c}\mathrm{C}_{22} \mathrm{H}_{17} \mathrm{~N}_{3} \mathrm{O}_{2} \\
(355.0)\end{array}$ & 174 & 72 & $\begin{array}{c}74.34 \\
(74.37)\end{array}$ & $\begin{array}{c}4.76 \\
(4.79)\end{array}$ & $\begin{array}{l}11.80 \\
(11.83)\end{array}$ \\
\hline $5 \mathrm{c}$. & $2-\mathrm{NO}_{2}$ & $\begin{array}{c}\mathrm{C}_{22} \mathrm{H}_{16} \mathrm{~N}_{4} \mathrm{O}_{3} \\
(384.0)\end{array}$ & 167 & 74 & $\begin{array}{c}68.71 \\
(68.75)\end{array}$ & $\begin{array}{c}4.16 \\
(4.17)\end{array}$ & $\begin{array}{c}14.56 \\
(14.58)\end{array}$ \\
\hline $5 \mathrm{~d}$. & 2-CI & $\begin{array}{c}\mathrm{C}_{22} \mathrm{H}_{16} \mathrm{~N}_{3} \mathrm{OCI} \\
(373.5)\end{array}$ & 162 & 68 & $\begin{array}{c}70.65 \\
(70.68)\end{array}$ & $\begin{array}{l}4.25 \\
(4.28)\end{array}$ & $\begin{array}{l}11.20 \\
(11.24)\end{array}$ \\
\hline $5 \mathrm{e}$. & $3-\mathrm{Br}$ & $\begin{array}{c}\mathrm{C}_{22} \mathrm{H}_{16} \mathrm{~N}_{3} \mathrm{OBr} \\
(418.0)\end{array}$ & 195 & 70 & $\begin{array}{c}63.15 \\
(63.16)\end{array}$ & $\begin{array}{c}3.79 \\
(3.83)\end{array}$ & $\begin{array}{c}10.02 \\
(10.05)\end{array}$ \\
\hline $5 \mathrm{f}$. & 4-CI & $\begin{array}{c}\mathrm{C}_{22} \mathrm{H}_{16} \mathrm{~N}_{3} \mathrm{OCl} \\
(373.5)\end{array}$ & 166 & 66 & $\begin{array}{l}70.65 \\
(70.68)\end{array}$ & $\begin{array}{c}4.26 \\
(4.28)\end{array}$ & $\begin{array}{l}11.21 \\
(11.24)\end{array}$ \\
\hline $5 \mathrm{~g}$. & $2-\mathrm{OCH}_{3}$ & $\begin{array}{c}\mathrm{C}_{23} \mathrm{H}_{19} \mathrm{~N}_{3} \mathrm{O}_{2} \\
(369.0)\end{array}$ & 170 & 75 & $\begin{array}{c}74.77 \\
(74.80)\end{array}$ & $\begin{array}{c}5.12 \\
(5.15)\end{array}$ & $\begin{array}{c}11.37 \\
(11.38)\end{array}$ \\
\hline $5 \mathrm{~h}$. & $\begin{array}{c}3,4- \\
\left(\mathrm{OCH}_{3}\right)_{2}\end{array}$ & $\begin{array}{c}\mathrm{C}_{24} \mathrm{H}_{21} \mathrm{~N}_{3} \mathrm{O}_{3} \\
(399.0)\end{array}$ & 187 & 66 & $\begin{array}{l}72.16 \\
(72.18)\end{array}$ & $\begin{array}{c}5.22 \\
(5.26)\end{array}$ & $\begin{array}{c}10.51 \\
(10.53)\end{array}$ \\
\hline $5 \mathrm{i}$. & $4-\mathrm{OCH}_{3}$ & $\begin{array}{c}\mathrm{C}_{23} \mathrm{H}_{19} \mathrm{~N}_{3} \mathrm{O}_{2} \\
(369.0)\end{array}$ & 176 & 73 & $\begin{array}{l}74.78 \\
(74.80)\end{array}$ & $\begin{array}{c}5.12 \\
(5.15)\end{array}$ & $\begin{array}{l}11.36 \\
(11.38)\end{array}$ \\
\hline $5 \mathrm{j}$. & $\begin{array}{l}3,4,5- \\
\left(\mathrm{OCH}_{3}\right)_{3}\end{array}$ & $\begin{array}{c}\mathrm{C}_{25} \mathrm{H}_{23} \mathrm{~N}_{3} \mathrm{O}_{4} \\
(429.0)\end{array}$ & 198 & 70 & $\begin{array}{c}69.90 \\
(69.93)\end{array}$ & $\begin{array}{c}5.33 \\
(5.36)\end{array}$ & $\begin{array}{c}9.77 \\
(9.79)\end{array}$ \\
\hline
\end{tabular}

Table - 6 ANTIMICROBIAL DATA OF COMPOUNDS [1(a-j)]

\begin{tabular}{ccccc}
\hline & & \multicolumn{3}{c}{ Zone of Inhibition (m.m.) } \\
\cline { 3 - 5 } Compd. No. & $\mathrm{R}$ & $\begin{array}{c}\text { Staphylococcus aureus } \\
\text { (Antibacterial) }\end{array}$ & $\begin{array}{c}\text { Escherichia coli } \\
\text { (Antibacterial) }\end{array}$ & $\begin{array}{c}\text { Candela albicans } \\
\text { (Antifungal) }\end{array}$ \\
\hline 1 a. & $-\mathrm{H}$ & 12.0 & 10.0 & 8.0 \\
1 b. & $2-\mathrm{OH}$ & 11.0 & 10.0 & 7.0 \\
1 c. & $2-\mathrm{NO}_{2}$ & 9.0 & 9.0 & 5.0 \\
1 d. & $2-\mathrm{CI}$ & 8.0 & 11.0 & 6.0 \\
1 e. & $3-\mathrm{Br}$ & 7.0 & 8.0 & 4.0 \\
1 f. & $4-\mathrm{CI}$ & 11.0 & 12.0 & 8.0 \\
1 g. & $2-\mathrm{OCH}_{3}$ & 11.0 & 9.0 & 6.0 \\
1 h. & $3,4-$ & 9.0 & 10.0 & 7.0 \\
1 i. & $\left.4-\mathrm{OCH}_{3}\right)_{2}$ & 10.0 & 10.0 & 6.0 \\
1 j. & $3,4,5-$ & 7.0 & 9.0 & 4.0 \\
\hline
\end{tabular}


Table - 7 ANTIMICROBIAL DATA OF COMPOUNDS [2(a-j)]

\begin{tabular}{|c|c|c|c|c|}
\hline \multirow{2}{*}{$\begin{array}{l}\text { Compd. } \\
\text { No. }\end{array}$} & \multirow{2}{*}{$\mathrm{R}$} & \multicolumn{3}{|c|}{ Zone of Inhibition (m.m.) } \\
\hline & & $\begin{array}{c}\text { Staphylococcus aureus } \\
\text { (Antibacterial) }\end{array}$ & $\begin{array}{l}\text { Escherichia coli } \\
\text { (Antibacterial) }\end{array}$ & $\begin{array}{c}\text { Candela albicans } \\
\text { (Antifungal) }\end{array}$ \\
\hline 2 a. & $-\mathrm{H}$ & 11.0 & 12.0 & 7.0 \\
\hline $2 \mathrm{~b}$. & $2-0 \mathrm{H}$ & 9.0 & 8.0 & 5.0 \\
\hline $2 \mathrm{c}$. & $2-\mathrm{NO}_{2}$ & 11.0 & 11.0 & 8.0 \\
\hline $2 \mathrm{~d}$ & 2-CI & 7.0 & 11.0 & 5.0 \\
\hline $2 \mathrm{e}$. & $3-\mathrm{Br}$ & 10.0 & 8.0 & 6.0 \\
\hline $2 \mathrm{f}$ & 4-CI & 12.0 & 10.0 & 8.0 \\
\hline $2 \mathrm{~g}$. & $2-\mathrm{OCH}_{3}$ & 8.0 & 7.0 & 4.0 \\
\hline $2 \mathrm{~h}$. & $\begin{array}{c}3,4- \\
\left(\mathrm{OCH}_{3}\right)_{2}\end{array}$ & 11.0 & 10.0 & 7.0 \\
\hline 2 i. & $4-\mathrm{OCH}_{3}$ & 9.0 & 8.0 & 5.0 \\
\hline $2 \mathrm{j}$. & $\begin{array}{l}3,4,5- \\
\left(\mathrm{OCH}_{3}\right)_{3} \\
\end{array}$ & 12.0 & 9.0 & 8.0 \\
\hline
\end{tabular}

Table - 8 ANTIMICROBIAL DATA OF COMPOUNDS [3(a-j)]

\begin{tabular}{|c|c|c|c|c|}
\hline \multirow{2}{*}{$\begin{array}{l}\text { Compd. } \\
\text { No. }\end{array}$} & \multirow{2}{*}{$\mathrm{R}$} & \multicolumn{3}{|c|}{ Zone of Inhibition (m.m.) } \\
\hline & & $\begin{array}{c}\text { Staphylococcus aureus } \\
\text { (Antibacterial) }\end{array}$ & $\begin{array}{c}\text { Escherichia coli } \\
\text { (Antibacterial) }\end{array}$ & $\begin{array}{c}\text { Candela albicans } \\
\text { (Antifungal) }\end{array}$ \\
\hline 3 a. & $-\mathrm{H}$ & 7.0 & 8.0 & 5.0 \\
\hline $3 \mathrm{~b}$. & $2-0 \mathrm{H}$ & 8.0 & 11.0 & 7.0 \\
\hline $3 \mathrm{c}$. & $2-\mathrm{NO}_{2}$ & 10.0 & 12.0 & 8.0 \\
\hline $3 \mathrm{~d}$. & 2-CI & 10.0 & 10.0 & 6.0 \\
\hline 3 e. & $3-\mathrm{Br}$ & 11.0 & 8.0 & 7.0 \\
\hline $3 \mathrm{f}$. & 4-CI & 12.0 & 11.0 & 8.0 \\
\hline $3 \mathrm{~g}$. & $2-\mathrm{OCH}_{3}$ & 9.0 & 7.0 & 5.0 \\
\hline $3 \mathrm{~h}$. & $\begin{array}{c}3,4- \\
\left(\mathrm{OCH}_{3}\right)_{2}\end{array}$ & 9.0 & 9.0 & 6.0 \\
\hline 3 i. & $4-\mathrm{OCH}_{3}$ & 10.0 & 12.0 & 8.0 \\
\hline $3 \mathrm{j}$ & $\begin{array}{l}3,4,5- \\
\left(\mathrm{OCH}_{3}\right)_{3}\end{array}$ & 7.0 & 7.0 & 4.0 \\
\hline
\end{tabular}


Table - 9 ANTIMICROBIAL DATA OF COMPOUNDS [4(a-j)]

\begin{tabular}{ccccc}
\hline & & \multicolumn{3}{c}{ Zone of Inhibition (m.m.) } \\
\cline { 3 - 5 } Compd. No. & $\mathrm{R}$ & $\begin{array}{c}\text { Staphylococcus aureus } \\
\text { (Antibacterial) }\end{array}$ & $\begin{array}{c}\text { Escherichia coli } \\
\text { (Antibacterial) }\end{array}$ & $\begin{array}{c}\text { Candela albicans } \\
\text { (Antifungal) }\end{array}$ \\
\hline 4 a. & $-\mathrm{H}$ & 9.0 & 8.0 & 5.0 \\
4 b. & $2-\mathrm{OH}$ & 10.0 & 8.0 & 7.0 \\
4 c. & $2-\mathrm{NO}_{2}$ & 12.0 & 11.0 & 8.0 \\
4 d. & $2-\mathrm{CI}$ & 8.0 & 7.0 & 4.0 \\
4 e. & $3-\mathrm{Br}$ & 10.0 & 12.0 & 7.0 \\
4 f. & $4-\mathrm{CI}$ & 7.0 & 8.0 & 4.0 \\
4 g. & $2-\mathrm{OCH}_{3}$ & 11.0 & 11.0 & 8.0 \\
4 h. & $3,4-$ & 9.0 & 10.0 & 6.0 \\
4 i. & $\left.4-\mathrm{OCH}_{3}\right)_{2}$ & 8.0 & 8.0 & 5.0 \\
4 j. & $3,4,5-$ & 12.0 & 10.0 & 8.0 \\
\hline
\end{tabular}

Table - 10 ANTIMICROBIAL DATA OF COMPOUNDS [5(a-j)]

\begin{tabular}{|c|c|c|c|c|}
\hline \multirow{2}{*}{$\begin{array}{l}\text { Compd. } \\
\text { No. }\end{array}$} & \multirow{2}{*}{$\mathrm{R}$} & \multicolumn{3}{|c|}{ Zone of Inhibition (m.m.) } \\
\hline & & $\begin{array}{c}\text { Staphylococcus aureus } \\
\text { (Antibacterial) }\end{array}$ & $\begin{array}{l}\text { Escherichia coli } \\
\text { (Antibacterial) }\end{array}$ & $\begin{array}{l}\text { Candela albicans } \\
\text { (Antifungal) }\end{array}$ \\
\hline 5 a. & $-\mathrm{H}$ & 12.0 & 12.0 & 8.0 \\
\hline $5 \mathrm{~b}$. & $2-0 \mathrm{H}$ & 8.0 & 7.0 & 4.0 \\
\hline $5 \mathrm{c}$. & $2-\mathrm{NO}_{2}$ & 11.0 & 10.0 & 7.0 \\
\hline $5 \mathrm{~d}$. & 2-CI & 9.0 & 8.0 & 5.0 \\
\hline 5 e. & $3-\mathrm{Br}$ & 10.0 & 10.0 & 6.0 \\
\hline $5 \mathrm{f}$. & 4-CI & 8.0 & 11.0 & 5.0 \\
\hline $5 \mathrm{~g}$. & $2-\mathrm{OCH}_{3}$ & 10.0 & 12.0 & 8.0 \\
\hline $5 \mathrm{~h}$. & $\begin{array}{c}3,4- \\
\left(\mathrm{OCH}_{3}\right)_{2}\end{array}$ & 7.0 & 7.0 & 4.0 \\
\hline 5 i. & $4-\mathrm{OCH}_{3}$ & 12.0 & 9.0 & 8.0 \\
\hline $5 \mathrm{j}$. & $\begin{array}{l}3,4,5- \\
\left(\mathrm{OCH}_{3}\right)_{3}\end{array}$ & 9.0 & 9.0 & 7.0 \\
\hline
\end{tabular}




\section{Materials and Methods}

All melting points were determined in open capillaries in a liquid paraffin bath and are uncorrected. The I.R. spectra were recorded with KBr pellets on Shimadzu FT-IR 8300 spectrophotometer and ${ }^{1} \mathrm{H}$ N.M.R. spectra were recorded on a Varian Geminy $200 \mathrm{MHz}$ spectrophotometer with $\mathrm{CDCl}_{3}$ / DMSO$\mathrm{d}_{6}$ as a solvent using tetramethylsilane (T.M.S.) as an internal standard; the chemical shift values are in $\delta \mathrm{ppm}$. The purity of the compounds was checked by thin layer chromatography (T.L.C.) on silica gel coated glass plates. The elemental analysis (i.e. C, $\mathrm{H}$ and $\mathrm{N}$ analysis) has been done on Perkin - Elmer model $240 \mathrm{~B} C H N$ analyzer and the values are within the permissible limits (i.e. \pm 0.5 ) of their calculated values.

\section{Antimicrobial Activity}

Antimicrobial activity of newly synthesised compounds was studied against gram-positive bacteria "Staphylococcus aureus" and gram-negative bacteria "Escherichia coli" (for antibacterial activity) and against the culture "Candela albicans" (for antifungal activity). The antimicrobial screening was carried out by cup - plate method ${ }^{\mathbf{1 0}}$ at a concentration of $50 \mu \mathrm{g} / \mathrm{mL}$ in solvent D.M.F. The zone of inhibition was measured in $\mathrm{mm}$. The antimicrobial activity of the synthesised compounds was compared with standard drugs Ampicillin, Penicillin and Tetracycline at the same concentration.

\section{Results and Discussion}

The antimicrobial activities of newly synthesised compounds were compared with known antibiotics like Ampicillin, Penicillin and Tetracycline and all the compounds show moderate to good activity. Structure elucidation of synthesised compounds has been made on the basis of elemental analysis, I.R. spectral studies and ${ }^{1}$ H N.M.R. spectral studies and all the compounds gave satisfactory elemental analysis, I.R. and ${ }^{1}$ H N.M.R. spectral measurements.

\section{I.R. Spectral Studies}

$>\quad$ I.R. $\left(\mathrm{cm}^{-1}\right)(\mathrm{KBr})$ spectral data of compound [1(j)]

$2834 \vee\left(\mathrm{C}-\mathrm{H}\right.$ stretching, $\mathrm{Ar}-\mathrm{OCH}_{3}$ at aryl methylidine ring); $1720 \vee(\mathrm{C}=\mathrm{O}$ stretching at oxazolone ring); $1598 \vee(\mathrm{C}=\mathrm{N}$ stretching, oxazolone ring $) ; 1555 \vee(\mathrm{C}=\mathrm{C}$ stretching at oxazolone ring $) ; 1333 \vee(\mathrm{C}-$ $\mathrm{H}$ bending, $\mathrm{Ar}-\mathrm{CH}_{3}$ at phenyl ring).

\section{$>$ I.R. $\left(\mathrm{cm}^{-1}\right)(\mathrm{KBr})$ spectral data of compound [2(e)]}

$1718 \vee(\mathrm{C}=\mathrm{O}$ stretching at imidazolinone ring $) ; 1592 \mathrm{v}(\mathrm{C}=\mathrm{N}$ stretching, imidazolinone ring); $1565 \mathrm{v}$ $(\mathrm{C}=\mathrm{C}$ stretching at imidazolinone ring $) ; 1375 \mathrm{v}\left(\mathrm{S}(=\mathrm{O})_{2}\right.$ stretching, $\left.\mathrm{Ar}-\mathrm{SO}_{2}-\mathrm{Ar}\right) ; 1326 \mathrm{v}(\mathrm{C}-\mathrm{H}$ bending, $\mathrm{Ar}-\mathrm{CH}_{3}$ at phenyl ring); $556 \mathrm{v}$ (C-Br stretching, $\mathrm{Ar}-\mathrm{Br}$ at aryl methylidine ring).

$>$ I.R. $\left(\mathrm{cm}^{-1}\right)(\mathrm{KBr})$ spectral data of compound $[3(\mathrm{~d})]-$

$1718 \vee(\mathrm{C}=\mathrm{O}$ stretching at imidazolinone ring); $1601 \vee(\mathrm{C}=\mathrm{N}$ stretching, imidazolinone ring $) ; 1565 \mathrm{v}$ $\left(\mathrm{C}=\mathrm{C}\right.$ stretching at imidazolinone ring); 1466 v $\left(\mathrm{C}-\mathrm{H}\right.$ bending, $\left.\mathrm{Ar}-\mathrm{CH}_{2}-\mathrm{Ar}\right) ; 1314 \mathrm{v}(\mathrm{C}-\mathrm{H}$ bending, Ar$\mathrm{CH}_{3}$ at phenyl ring); $739 \mathrm{v}(\mathrm{C}-\mathrm{Cl}$ stretching, $\mathrm{Ar}-\mathrm{Cl}$ at aryl methylidine ring).

\section{$>$ I.R. $\left(\mathrm{cm}^{-1}\right)(\mathrm{KBr})$ spectral data of compound [4(a)]}

$1708 \mathrm{v}(\mathrm{C}=\mathrm{O}$ stretching at imidazolinone ring); $1604 \mathrm{v}(\mathrm{C}=\mathrm{N}$ stretching, imidazolinone ring $) ; 1560 \mathrm{v}$ $(\mathrm{C}=\mathrm{O}$ stretching, Ar-CONH-Ar); $1554 \vee(\mathrm{C}=\mathrm{C}$ stretching at imidazolinone ring $) ; 1326 \vee(\mathrm{C}-\mathrm{H}$ bending, $\mathrm{Ar}-\mathrm{CH}_{3}$ at phenyl ring).

$>\quad$ I.R. $\left(\mathrm{cm}^{-1}\right)(\mathrm{KBr})$ spectral data of compound $[5(\mathrm{c})]$ :-

$1718 \vee(\mathrm{C}=\mathrm{O}$ stretching at imidazolinone ring); $1602 \vee(\mathrm{C}=\mathrm{N}$ stretching, imidazolinone ring $) ; 1571 \mathrm{v}$ $\left(\mathrm{C}=\mathrm{C}\right.$ stretching at imidazolinone ring); $1518 \vee\left(\mathrm{N}=\mathrm{O}\right.$ stretching, $\mathrm{Ar}-\mathrm{NO}_{2}$ at aryl methylidine ring); $1357 v\left(\mathrm{C}-\mathrm{N}\right.$ stretching); $1321 \mathrm{v}\left(\mathrm{C}-\mathrm{H}\right.$ bending, $\mathrm{Ar}-\mathrm{CH}_{3}$ at phenyl ring). 
${ }^{I} H$ N.M.R. Spectral Studies

${ }^{1} \mathrm{H}$ N.M.R. ( $\left.\mathrm{CDCl}_{3}\right)$ spectral data of compound [1(c)]

$2.47 \delta \mathrm{ppm}$ (s, $3 \mathrm{H}, \mathrm{Ar}-\mathrm{CH}_{3}$ at phenyl ring); $5.64 \delta \mathrm{ppm}(\mathrm{s}, 1 \mathrm{H}, \mathrm{Ar}=\mathrm{CH}-$ at oxazolone ring); 7.13 to $8.22 \delta \mathrm{ppm}(\mathrm{m}, 8 \mathrm{H}, \mathrm{Ar}-\mathrm{H})$.

$>{ }^{1} \mathrm{H}$ N.M.R. $\left(\mathrm{CDCl}_{3}+\right.$ DMSO-d $\left.\mathrm{d}_{6}\right)$ spectral data of compound [2(d)]

$2.44 \delta \mathrm{ppm}\left(\mathrm{s}, 6 \mathrm{H}, 2 \times \mathrm{Ar}-\mathrm{CH}_{3}\right.$ at phenyl ring); $5.62 \delta \mathrm{ppm}(\mathrm{s}, 2 \mathrm{H}, 2 \times \mathrm{Ar}=\mathrm{CH}-$ at imidazolinone ring); 7.15 to $8.21 \delta \mathrm{ppm}(\mathrm{m}, 24 \mathrm{H}, \mathrm{Ar}-\mathrm{H})$.

$>{ }^{1} \mathrm{H}$ N.M.R. $\left(\mathrm{CDCl}_{3}+\right.$ DMSO-d $\left.\mathrm{d}_{6}\right)$ spectral data of compound [3(e)]

$2.49 \delta \mathrm{ppm}\left(\mathrm{s}, 6 \mathrm{H}, 2 \times \mathrm{Ar}-\mathrm{CH}_{3}\right.$ at phenyl ring); $2.92 \delta \mathrm{ppm}\left(\mathrm{s}, 2 \mathrm{H}, \mathrm{Ar}-\mathrm{CH}_{2}-\mathrm{Ar}\right) ; 5.48 \delta \mathrm{ppm}(\mathrm{s}, 2 \mathrm{H}$, $2 \times \mathrm{Ar}=\mathrm{CH}-$ at imidazolinone ring); 7.17 to $8.25 \delta \mathrm{ppm}(\mathrm{m}, 24 \mathrm{H}, \mathrm{Ar}-\mathrm{H})$.

$>{ }^{1} \mathrm{H}$ N.M.R. $\left(\mathrm{CDCl}_{3}\right)$ spectral data of compound [4(a)]

$2.51 \delta \mathrm{ppm}\left(\mathrm{s}, 6 \mathrm{H}, 2 \times \mathrm{Ar}^{-\mathrm{CH}_{3}}\right.$ at phenyl ring); $5.65 \delta \mathrm{ppm}(\mathrm{s}, 2 \mathrm{H}, 2 \times \mathrm{Ar}=\mathrm{CH}-$ at imidazolinone ring); 7.12 to $8.13 \delta \mathrm{ppm}(\mathrm{m}, 24 \mathrm{H}, \mathrm{Ar}-\mathrm{H}) ; 8.76 \delta \mathrm{ppm}(\mathrm{s}, 1 \mathrm{H}, \mathrm{Ar}-\mathrm{CONH}-\mathrm{Ar})$.

$>{ }^{1} \mathrm{H}$ N.M.R. $\left(\mathrm{CDCl}_{3}\right)$ spectral data of compound [5(f)]

$2.52 \delta \mathrm{ppm}\left(\mathrm{s}, 3 \mathrm{H}, \mathrm{Ar}-\mathrm{CH}_{3}\right.$ at phenyl ring); $5.63 \delta \mathrm{ppm}(\mathrm{s}, 1 \mathrm{H}, \mathrm{Ar}=\mathrm{CH}-$ at imidazolinone ring); 7.10 to $8.14 \delta \mathrm{ppm}(\mathrm{m}, 12 \mathrm{H}, \mathrm{Ar}-\mathrm{H})$.

\section{Acknowledgements}

The authors are thankful to the Department of Chemistry, Veer Narmad South Gujarat University, Surat, for providing necessary laboratory facilities. They are also grateful to the Department of Biosciences, Veer Narmad South Gujarat University, Surat, for screening the newly synthesised compounds for their antimicrobial activities; Wyeth Lederle Limited, Atul, for scanning the I.R. spectra and Department of Central Instrumentation Laboratory, Punjab University, Chandigarh, for screening the ${ }^{1}$ H N.M.R. spectra of newly synthesized compounds.

\section{References}

1. Neimann C and Redemann C A, J Am Chem So, 1949, 63, 1549.

2. The Chemistry of the Antibiotics used in Medicine; Peragamon Publication by Evans, 1965.

3. Brink and Harman; Quart. Rev., 1958, 12, 93.

4. Barry, Quart. Rev., 1966, 20, 559.

5. Cornforth J W, in Clarke H T, Johnson J R and Robinson R, "Chemistry of Penicillin", Princeton University Press, Princeton, New Jercey, 1949, p.778.

6. Agarwal R, Chaudhary C and Mishra V, Ind J Chem, 1983, 22(B), 308.

7. Brown T H, Chem Abst, 1984, 102, 16649a.

8. $\quad$ Karjalainen A. Kurkel K and Seppo A, Eur Pat, 58047.

9. Nalthani P K, Srivastava V K and Shankar A K, Ind J Chem., 1989, 28(B), 990.

10. Barry A L, in : Illus (Ed.), The Antimicrobial Susceptibility Test: Principle and Practices, Lea and Febiger, Philadelphia, Pa, U.S.A., 1976, p.180; Biol. Abstr., 1977, 64, 25183. 


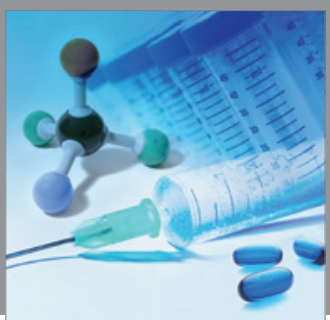

International Journal of

Medicinal Chemistry

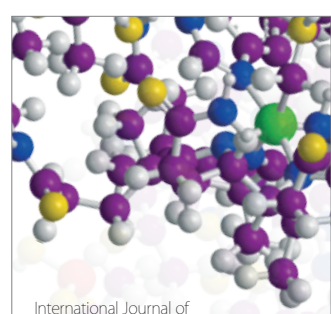

Carbohydrate Chemistry

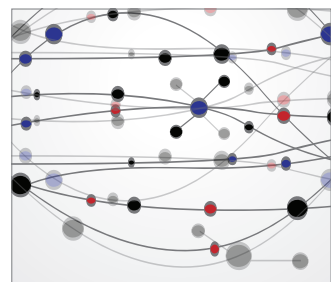

The Scientific World Journal
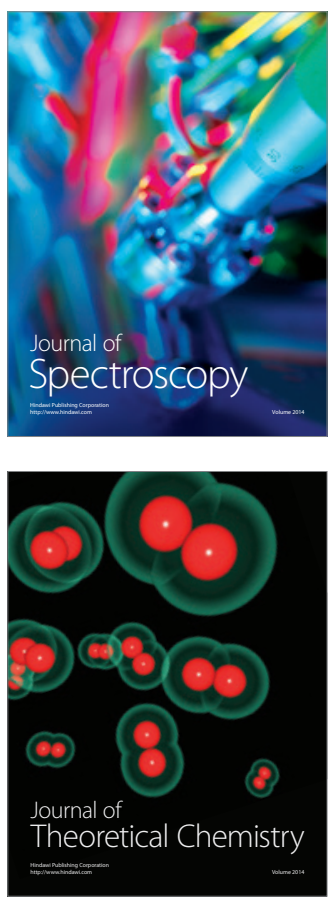
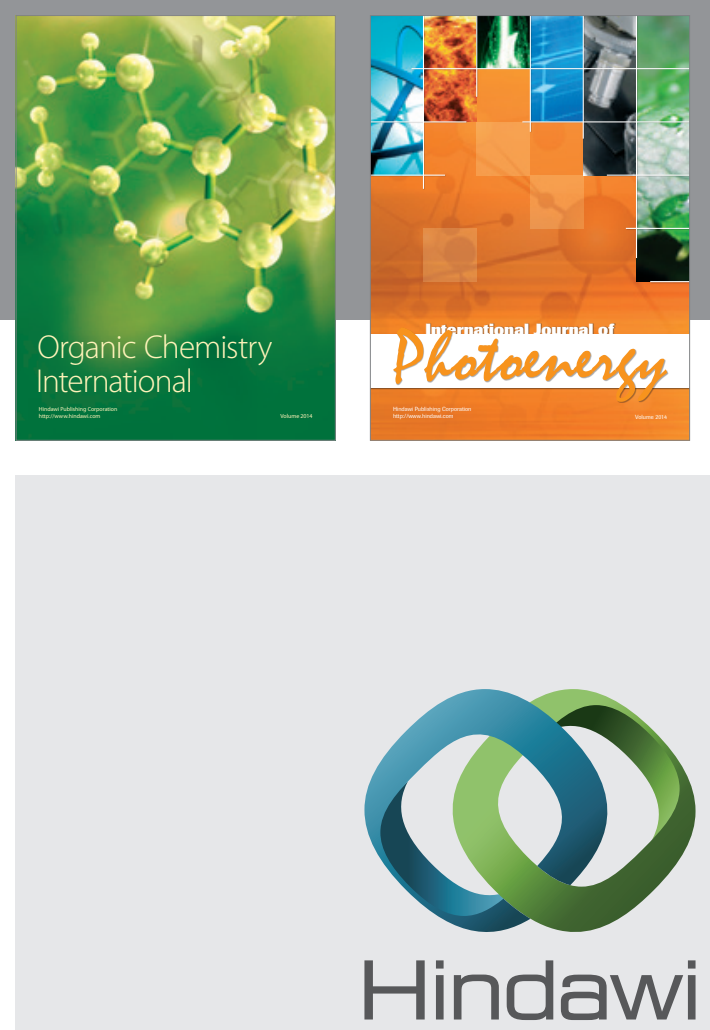

Submit your manuscripts at

http://www.hindawi.com
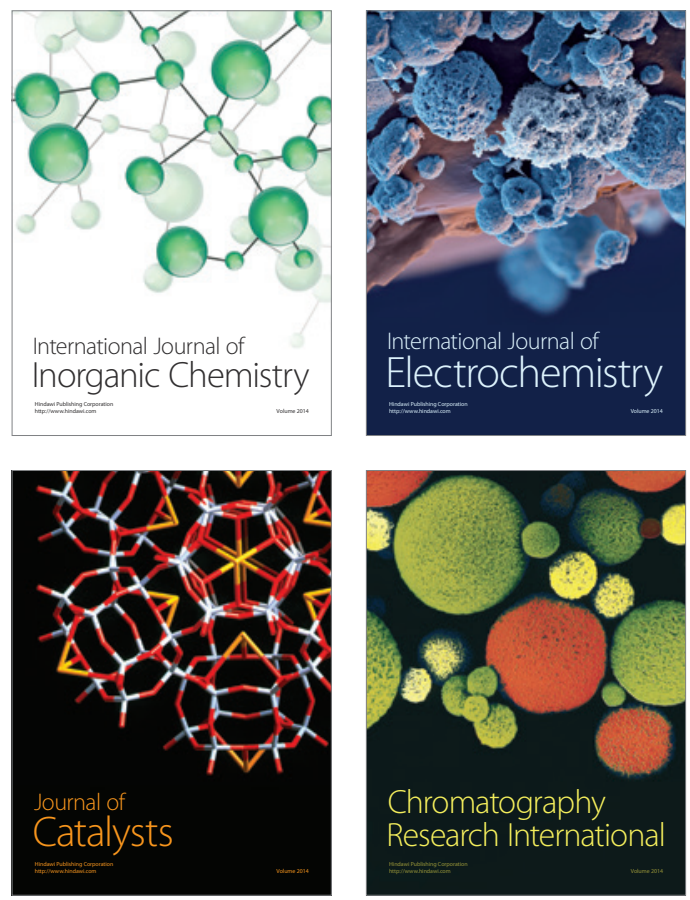
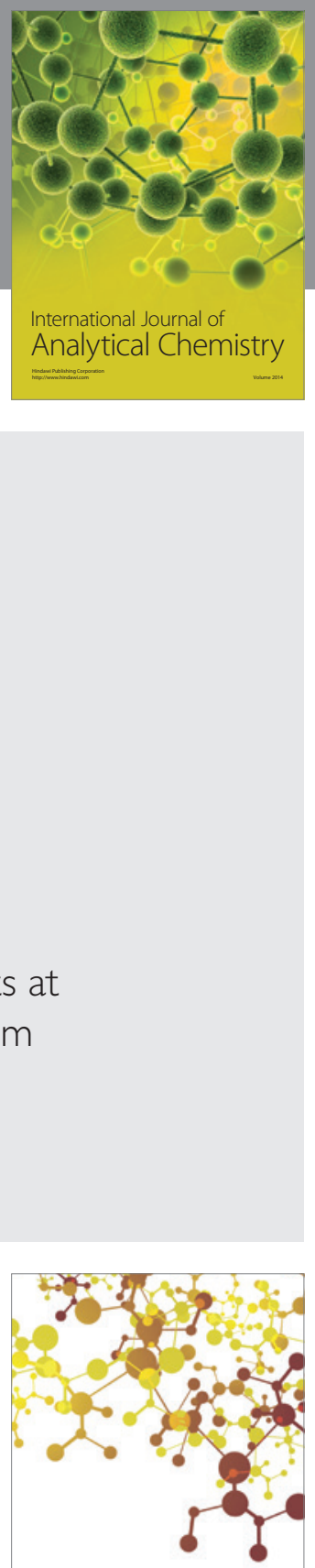

Journal of

Applied Chemistry
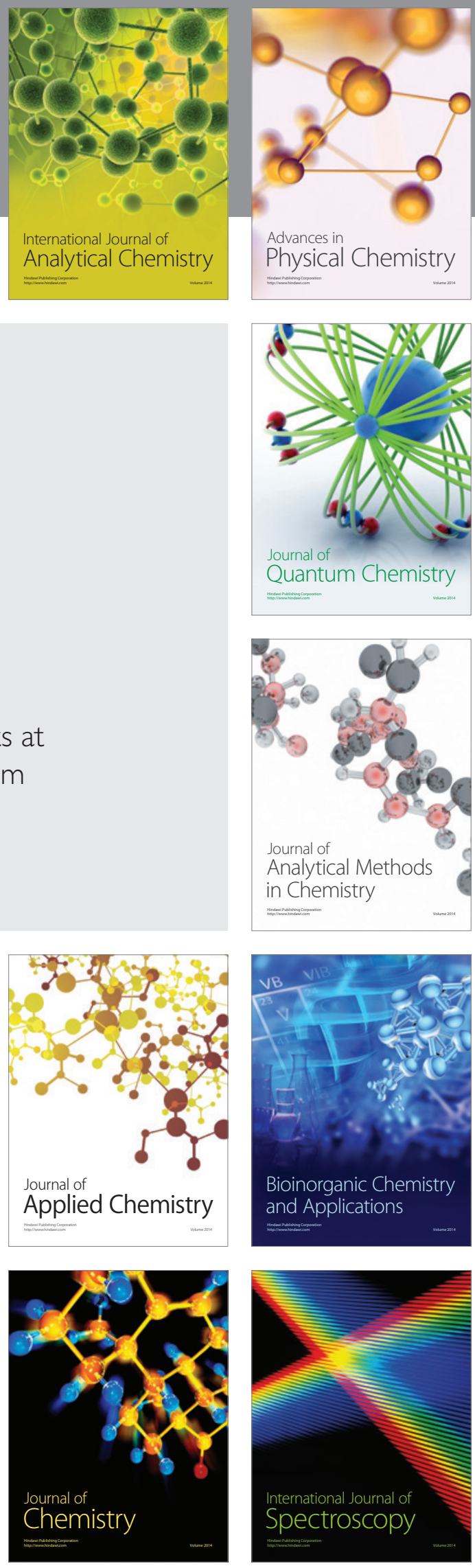